\title{
Rare submandibular gland mammary analogue secretory carcinoma: a case report
}

PY Wong ${ }^{1}$, D Al-Okati², H Kaddour ${ }^{1}$, G Kumar ${ }^{1}$

${ }^{1}$ Department of Ear, Nose and Throat Surgery, Queen's Hospital, Romford, UK

${ }^{2}$ Department of Pathology, Queen's Hospital, Romford, UK

\section{Background}

Mammary analogue secretory carcinoma (MASC) is a rare malignant salivary gland tumour, predominantly arises from the parotid gland. To date, there has been only 6 cases of submandibular gland MASC reported worldwide ${ }^{1}$. We report a case of a young male with submandibular gland MASC.

\section{Method}

Retrospective review of the clinical history, examination, investigation and management of this submandibular gland MASC case.

\section{Case History}

We present a 16 year old male who presented to his GP with epistaxis but was found to have a left submandibular lump. Patient gives a 5 year history of gradual increase in size of the lump but otherwise asymptomatic. Two years prior, he was seen by a Maxillofacial surgeon and was reassured that the lump was benign. He was lost to follow up.

On examination, the lump is well defined and mobile. All other ENT examinations were normal.

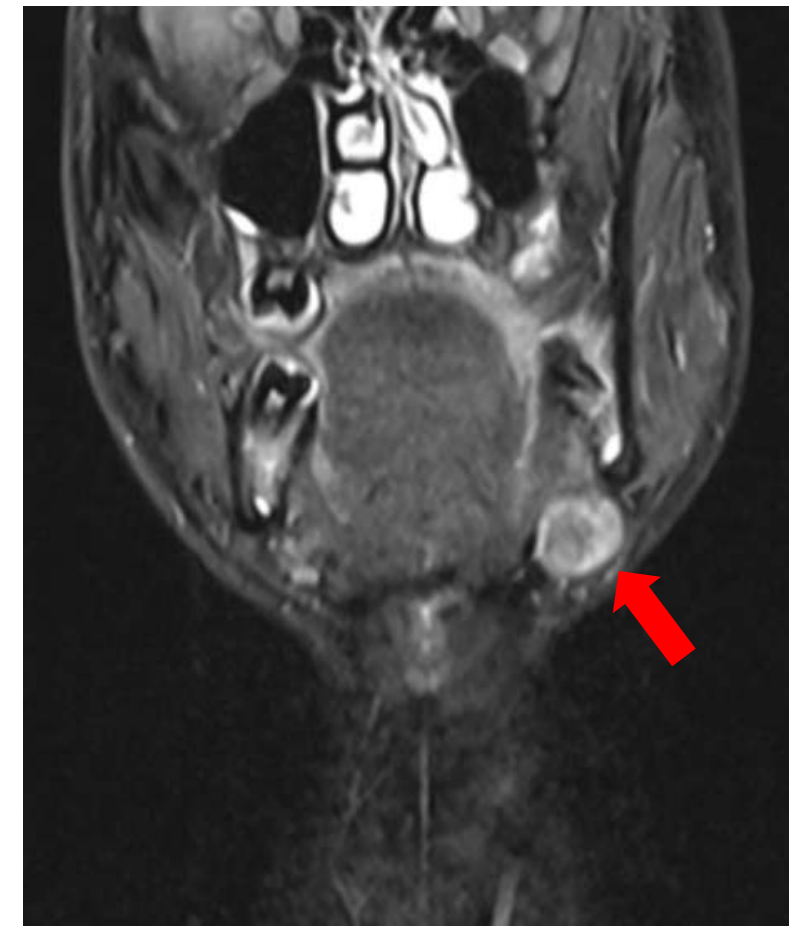

Figure 1: Coronal view of magnetic resonance imaging of the neck: Red arrow pointing to left submandibular gland tumour $(2.5 \times 1.5 \mathrm{~cm})$

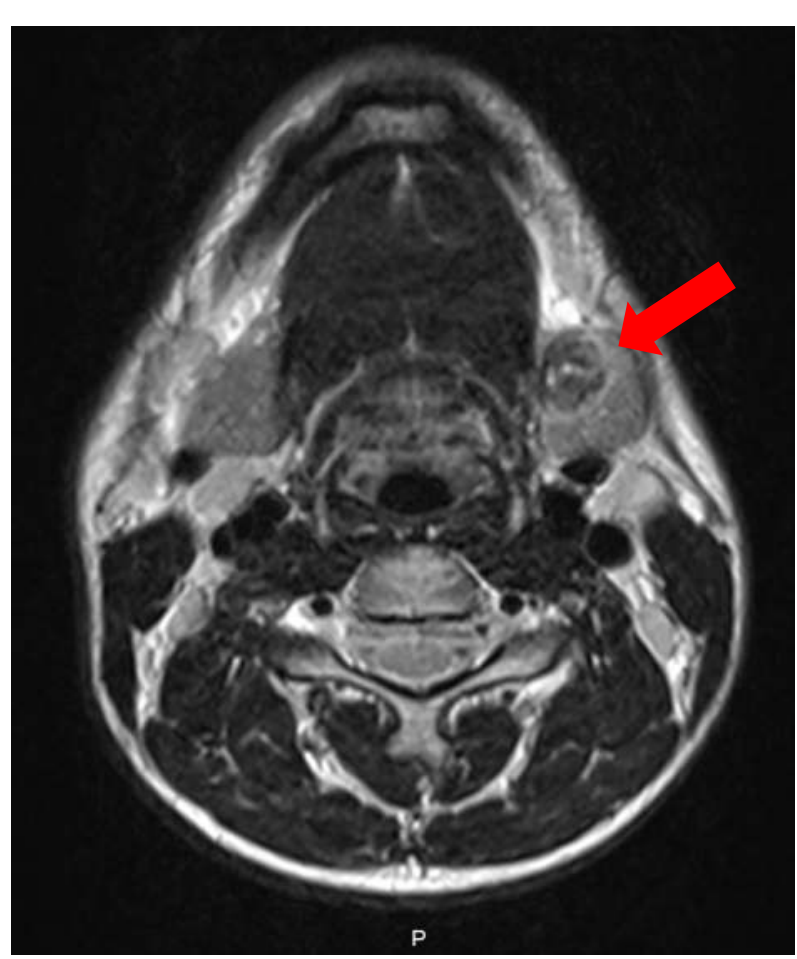

Figure 2: Axial view of magnetic resonance imaging of the neck; Red arrow pointing to left submandibular gland tumour

Ultrasound and magnetic resonance imaging (MRI) scan reported features suggestive of pleomorphic adenoma. He underwent routine uncomplicated excision of left submandibular gland. He was later referred Head and Neck multidisciplinary meeting as histology showed MASC.

\section{Discussion}

Patient had delay in treatment as was initially thought to be a benign tumour. The diagnosis came to light after excision of the gland. There is no local or regional recurrence detected at 1 year follow up.

\section{Conclusion}

The possibility of salivary MASC should always be considered in a young patient presenting with slow growing painless submandibular gland lump. 